\title{
Release of Oxytocin in the Rat Central Amygdala Modulates Stress-Coping Behavior and the Release of Excitatory Amino Acids
}

\author{
Karl Ebner*,', Oliver J Bosch², Simone A Krömer ${ }^{3}$, Nicolas Singewald' and Inga D Neumann $^{2}$ \\ 'Department of Pharmacology and Toxicology and Center for Molecular Biosciences Innsbruck (CMBI), University of Innsbruck, Innsbruck, Austria; \\ ${ }^{2}$ Institute of Zoology, University of Regensburg, Regensburg, Germany; ${ }^{3}$ Max Planck Institute of Psychiatry, Munich, Germany
}

\begin{abstract}
Previous experiments have indicated that the release of oxytocin (OXT) occurs in various hypothalamic and extrahypothalamic brain areas. In the present study, we investigated in male rats whether swim stress triggers the release of OXT in the central amygdala (CeA), a key area in processing emotions and stress responses. Further, we examined the physiological significance of OXT released within the CeA for behavioral responses during forced swimming as well as effects on the local release of selected amino acids including glutamate, aspartate, arginine, taurine, and GABA, which are thought to modulate processing of emotions. Exposure to a 10 -min forced swimming session caused a significant increase in OXT release $(200 \%, p<0.0 \mathrm{I})$ within, but not outside, the CeA as monitored by microdialysis. Administration of the OXT receptor antagonist des- $\mathrm{Gly}-\mathrm{NH}_{2} d\left(\mathrm{CH}_{2}\right)_{5}\left(\mathrm{Tyr}(\mathrm{Me})^{2} \mathrm{Thr}^{4}\right) \mathrm{OVT}$ via inverse microdialysis into the amygdala before and during exposure to swimming reduced the floating time by $55 \%(p<0.05)$ and increased the swimming time by $29 \%$ $(p<0.05)$ indicative of a more active stress-coping strategy. Simultaneously, local administration of the OXT receptor antagonist caused a significant increase in the stress-induced release of the excitatory amino acids glutamate and aspartate, whereas the basal release of these amino acids remained unchanged. Taken together, these findings demonstrate a significant activation of the oxytocinergic system in the $\mathrm{CeA}$ in response to swim stress. Furthermore, our data indicate that OXT receptor-mediated mechanisms within the amygdala are involved in the generation of passive stress-coping strategies, which might be mediated at least in part via its inhibitory influence on the local release of excitatory amino acids during stress.
\end{abstract}

Neuropsychopharmacology (2005) 30, 223-230, advance online publication, 10 November 2004; doi: I 0. I 038/sj.npp. I 300607

Keywords: stress; amygdala; oxytocin; coping strategy; forced swimming; amino acids

\section{INTRODUCTION}

The neuropeptide oxytocin (OXT) is well acknowledged to be involved in the regulation of physiological and behavioral functions related to female reproduction and affiliation at the brain level (Richard et al, 1991; Landgraf and Neumann, 2004), whereas its functions in the male brain are less well documented. There is, however, accumulating evidence that OXT released within various brain regions is a regulator of emotionality and physiological stress responses not only in the female rat (Windle et al, 1997, 2004; Neumann et al, 2000a, 2001; Lightman et al,

\footnotetext{
*Correspondence: Dr K Ebner, Leopold-Franzens-University of Innsbruck, Department of Pharmacology and Toxicology and Center for Molecular Biosciences Innsbruck (CMBI), Peter Mayr-Str. I, A-6020 Innsbruck, Austria, Tel: + 43512507 5623, Fax: + 435125072760 , E-mail: karl.ebner@uibk.ac.at

Received 17 June 2004; revised 23 September 2004; accepted 24 September 2004

Online publication: 6 October 2004 at http://www.acnp.org/citations/ Npp I00604040280/default.pdf
}

2001) but also in males as it plays an important role in cardiovascular (Callahan et al, 1989) and neuroendocrine (Neumann et al, 2000b) responses to an acute stressor. Stress exposure triggers not only OXT secretion into blood (Lang et al, 1983; Kasting, 1988; Wotjak et al, 1998) but also within the brain as reflected by increased OXT concentrations in the cerebrospinal fluid (Iványi et al, 1991) and extracellular space of several brain regions (for review, see Landgraf and Neumann, 2004). In more detail, it has been shown that psycho-social or physical stressors like social defeat, swim, or shaker stress evoke OXT release in various areas known to be involved in the modulation of stress mechanisms, including the hypothalamic supraoptic (SON) and paraventricular (PVN) nuclei where OXT neurons are localized as well as other limbic brain areas (Nishioka et al, 1998; Wotjak et al, 1998; Engelmann et al, 1999; Ebner et al, 2000; Wigger and Neumann, 2002; Bosch et al, 2004). The stress-induced patterns of local release of OXT within the brain, however, are dependent on various factors such as gender, type of stressor, and the genetically determined stress susceptibility. For example, our own investigations 
have shown that confrontation with an aggressive conspecific during social defeat-a psycho-social and emotional stressor - caused an increased release of OXT within the septum in male intruders defeated by a male resident (Ebner et al, 2000), but not in virgin female intruders defeated by a lactating resident (Bosch et al, 2004), whereas the same stressor induced a significant OXT release within the PVN of females (Bosch et al, 2004) but not of males (Wotjak et al, 1996).

The amygdala is a brain region particularly relevant for the processing of behavioral and neuroendocrine stress responses (Aggleton, 1992; Gray, 1996), in particular with respect to the oxytocinergic system (Neumann et al, 2000b; Bale et al, 2001). Within the amygdala, a substantial number of oxytocinergic fibers (Sofroniew, 1983) and OXT receptors have been localized mainly in the central (CeA) and medial amygdala (Barberis and Tribollet, 1996; Gimpl and Fahrenholz, 2001), suggesting that locally released OXT is a potential mediator of the complex stress response. Indeed, local blockade of OXT receptors within the amygdala resulted not only in altered emotionality (Bale et al, 2001; Neumann, 2002) but also in a disinhibition of the hypothalamo-pituitary-adrenal (HPA) axis of male rats (Neumann et al, 2000b). However, in contrast to the female (Bosch et al, 2004), the release of OXT within the amygdala has never been monitored in the male animal, which has also been partly due to the lower density of immunoreactive fibers and, consequently, to lower extracellular concentrations compared to hypothalamic areas.

On the basis of these observations, the intracerebral microdialysis experiments of this study were designed in order to investigate whether (i) OXT release within the CeA can also be monitored in the male rat, and (ii) exposure to a robust stressor like forced swimming triggers an increase in local OXT release within the CeA. Furthermore, we studied the physiological significance of locally released OXT within the CeA by administration of a selective OXT receptor antagonist bilaterally into the CeA using inverse microdialysis concomitantly with stress exposure, behavioral monitoring, and sampling of dialysates. We investigated whether OXT within the CeA is involved in the regulation (iii) of active or passive stress-coping style as reflected by respective behavioral parameters during forced swimming, and (iv) of the local release of relevant excitatory and inhibitory amino acids including glutamate, aspartate, taurine, and GABA. Since amino acids such as glutamate and GABA provide the principal sources of synaptic transmission in the amygdala (Davis et al, 1994; Maren, 1996; Sah et al, 2003), stress-induced changes in amygdala extracellular levels of these amino acids (Singewald et al, 2000) have been implicated in the modulation of emotional behavior (Davis et al, 1994).

\section{MATERIALS AND METHODS}

\section{Animals}

Experiments were performed on adult male Wistar rats (Charles River, Sulzfeld, Germany) weighing $300-400 \mathrm{~g}$ at the day of surgery. After delivery from the supplier, the animals were housed in groups of 4-6 in the animal house for at least 1 week. After surgery, they were kept individually in transparent polycarbon cages $(20 \times 28 \times 35 \mathrm{~cm})$ under controlled standard laboratory conditions $(12: 12 \mathrm{~h}$ light: dark cycle, lights on at 0700 hours, $22^{\circ} \mathrm{C}, 60 \%$ humidity) with food and water available ad libitum.

\section{Surgery}

All surgical, sampling, and behavioral procedures were approved by the Committee on Animal Health and Care of the local governmental administration. Under halothane (Hoechst, Frankfurt/Main, Germany) anesthesia, microdialysis probes (U-shaped membrane with a molecular cutoff of $18 \mathrm{kDa}$ ) were implanted stereotaxically into the CeA (Figure 1; implantation coordinates: $1.8 \mathrm{~mm}$ caudal to bregma, $3.8 \mathrm{~mm}$ lateral to the midline, and $9.4 \mathrm{~mm}$ deep from the surface of the skull) either unilaterally (experiment 1 ) or bilaterally (experiment 2) according to the stereotaxic atlas of Paxinos and Watson (1998). The probes were fixed to the skull with two jeweler's screws and dental cement. The two endings of each probe were connected to approximately $5 \mathrm{~cm}$ long pieces of PE-20 polyethylene tubing and fixed with dental cement. After surgery, the animals were injected with depot antibiotics $(0.04 \mathrm{ml} / \mathrm{rat}$ s.c.; Tardomycel, Bayer, Leverkusen, Germany) to prevent infections. On the next day, animals were handled for at least $5 \mathrm{~min}$ to familiarize them with the experimental equipment and to minimize nonspecific stress responses during the experiment.

\section{Microdialysis and Behavioral Procedure}

At 2 days after surgery, the microdialysis probes were perfused with sterile Ringer's solution (Fresenius, Bad Homburg, Germany) through the inflow line connected to a microinfusion pump at a flow rate of $3.3 \mu \mathrm{l} / \mathrm{min}$ for $2 \mathrm{~h}$ prior to the initiation of the experiment. During this period, sample collection was simulated every $30 \mathrm{~min}$ to adapt the animals to the microdialysis sampling.

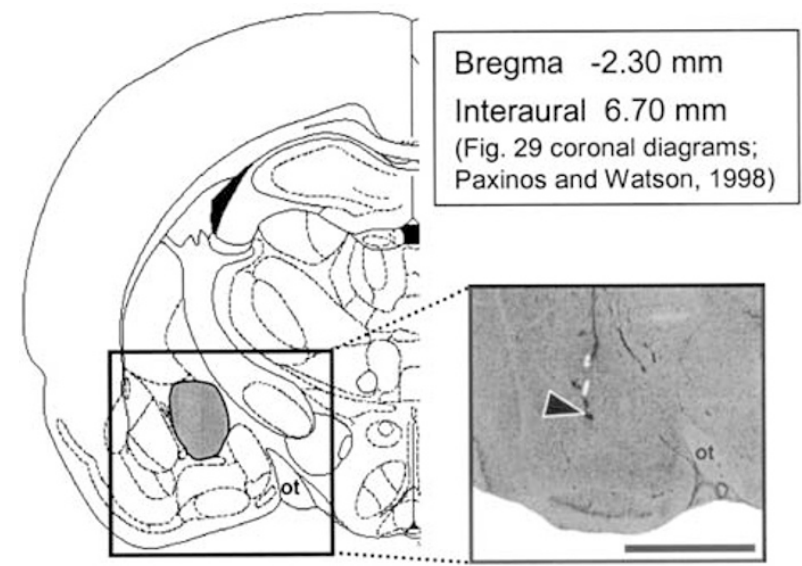

Figure I Schematic drawing (Paxinos and Watson, 1998) and a representative enlargement of a microphotograph of a cresyl-stained coronal section of the rat brain showing localization of the tip of the membrane of a microdialysis probe (arrowhead) within the CeA (gray shaded area). ot, optic tract. Scale bar, $250 \mu \mathrm{m}$. 
The forced swimming procedure has been described in detail previously (Ebner et al, 1999). Briefly, the rats were forced to swim for $10 \mathrm{~min}$ in a cylindrical Plexiglas tank (30 $\mathrm{cm}$ in diameter) filled to a depth of $40 \mathrm{~cm}$ with tap water $\left(20 \pm 1^{\circ} \mathrm{C}\right)$ during ongoing microdialysis. In experiment 2 , the behavior of the animals was monitored by a video system and scored by a trained observer blind to the animals' treatment. The duration in which the animals displayed one of the three following behaviors was measured by pressing preset keys on a PC that included an automatic timing process: (1) struggling, defined as movements during which the forelimbs broke the water's surface (eg scratching along the wall); (2) swimming, defined as movement of the animal induced by movements of the fore and hind limbs without breaking the water surface; and (3) floating defined as the behavior during which the animal used limb movement just to keep its equilibrium without any movement of the trunk.

\section{Experimental Protocols}

Experiment 1: Effects of forced swimming on the release of OXT within the amygdala. After termination of the initial $2 \mathrm{~h}$ perfusion period without sampling, five consecutive 30 min dialysates were collected directly into Eppendorf tubes containing $10 \mu \mathrm{l}$ of $0.1 \mathrm{~N} \mathrm{HCl}$, shock frozen, and stored at $-20^{\circ} \mathrm{C}$ until assay. Dialysates 1 and 2 were taken under basal (undisturbed) conditions. At the beginning of the third dialysis interval, animals $(n=13)$ were transferred from their home cages to the swim tank for 10 min during ongoing microdialysis, and thereafter they were gently dried using a towel and returned to their home cages. Dialysates 4 and 5 were again taken under undisturbed conditions.

Experiment 2: Effects of an OXT receptor antagonist within the amygdala on swim behavior and the local release of amino acids. In two additional groups of animals, microdialysis probes were implanted bilaterally into the CeA. After 2 days, five consecutive 30-min microdialysis samples were collected after an initial $2 \mathrm{~h}$ perfusion period as described in experiment 1 . During perfusion period 1 , animals of both groups were dialyzed bilaterally with Ringer's solution, and during perfusion period 2, either with Ringer's solution (controls, $n=5$ ) or Ringer's solution containing the OXT receptor antagonist des-Gly$\mathrm{NH}_{2} d\left(\mathrm{CH}_{2}\right)_{5}\left(\mathrm{Tyr}(\mathrm{Me})^{2} \mathrm{Thr}^{4}\right)$ OVT $(10 \mu \mathrm{g} / \mathrm{ml}$, Dr M Manning, Toledo, USA; $n=6$ ). At the beginning of the third dialysis interval, rats were forced to swim for $10 \mathrm{~min}$ under ongoing microdialysis without disconnecting them from the dialysis line, and their behavior was recorded by a video system and scored by a trained observer blind to the animals' treatment. After the 10-min stress exposure, the animals were returned to their home cages and microdialysis was further continued with Ringer's solution in all animals. During a 40-min retrodialysis period, an estimated amount of approximately $1.3 \mathrm{ng}$ antagonist was delivered into the surrounding extracellular space (Engelmann et al, 1992).

\section{Histology}

At the end of the experiment, animals were killed by an overdose of halothane. Brains were removed from the skull, shock frozen in dry ice chilled $n$-methylbutane (Carl Roth $\mathrm{GmbH}$, Karlsruhe, Germany), and stored at $-20^{\circ} \mathrm{C}$. For histological verification of the microdialysis probe placement, brains were sectioned in a cryocut (Microm HM 500, Walldorf, Germany) and $25 \mu \mathrm{m}$ coronal sections were stained with cresyl violet (Figure 1).

\section{Quantification of OXT and Amino Acids in Dialysates}

The concentration of OXT was measured in lyophilized microdialysates by a highly sensitive and selective radioimmunoassay (detection limit: $0.01 \mathrm{pg} / \mathrm{sample}$; crossreactivity of the antiserum with other related peptides, including arginine vasopressin, was less than $0.7 \%$ ) using synthetic OXT (Sigma, St Louis, MO, USA) as a standard and iodinated peptide (specific activity $2.200 \mathrm{Ci} / \mathrm{mmol}$; NEN, USA) as a tracer. The intra-assay coefficient of variation at relevant concentrations of the standard curve was between 7 and $10 \%$ (for a detailed description, see Landgraf et al, 1995).

In experiment 2, the amino-acid content in microdialysates collected from the left and right amygdala of each rat was determined separately by high-performance liquid chromatography (HPLC) and fluorimetric detection (Merck-Hitachi, Tokyo, Japan) after derivatization with $o$-phthaldialdehyde (OPA) as previously described (Singewald et al, 1995). In the present study, $75 \mu$ of a mixture of $80 \mu \mathrm{l}$ dialysate and $20 \mu \mathrm{l}$ OPA reaction mixture was injected. The reproducibility of the derivatization was controlled by addition of $S$-carboxymethyl-1-cysteine as an internal standard. Blank samples were treated identically and showed a negligible amount of the respective amino acid.

\section{Statistics}

Experimental subjects were included in the statistical analysis only if the microdialysis probes have been localized in the respective target brain area (Figure 1). Statistical analysis was performed using a computer software package (GB-Stat 6.0, Dynamic Microsystems, Silver Springs, USA). Raw data of the microdialysates were logarithmically transformed (to fit to Gaussian distribution) and submitted to a two-way ANOVA with repeated measures (group $\times$ time) followed by a Newman-Keuls (OXT, experiment 1 ) or Fisher's LSD post hoc (amino acids, experiment 2) analysis. For the statistical analysis of the amino acids in microdialysates sampled bilaterally from the left and right amygdala, we used the average of the two samples if both probes were placed correctly, or the data from the correctly placed probe. Behavioral measures were tested by MannWhitney U-test. Data are presented as means \pm SEM. Significance was accepted if $p<0.05$.

\section{RESULTS}

Histological analysis revealed that in nine out of 13 rats of experiment 1 and in all 11 rats of experiment 2 , the implanted microdialysis probes were localized within the CeA (Figure 1). Misplaced probes were localized dorsal $(n=3)$, or dorsal and anterior to the amygdala $(n=1)$. One control animal of experiment 2 had missing values and was therefore not included in the statistical analysis. 
Experiment 1: Effects of forced swimming on the release of OXT within the amygdala. As shown in Figure 2, forced swimming caused a significant increase in the OXT content of microdialysates collected from the CeA, which remained significantly elevated up to $60 \mathrm{~min}$ after the onset of the stressor (two-way ANOVA, factor group $\times$ time; $\mathrm{F}_{4,44}=3.09$, $p=0.02$ ). In contrast, OXT content in microdialysates sampled outside the amygdala remained at prestress levels throughout the dialysis period (Figure 2).

Experiment 2: Effects of an OXT receptor antagonist within the amygdala on swim behavior and the local release of amino acids. Administration of the OXT receptor antagonist bilaterally into the CeA significantly affected the animals' behavior during forced swimming. As illustrated in Figure 3, animals treated with the OXT receptor antagonist showed a reduced floating behavior $(p=0.02)$ and an increased time spent swimming $(p=0.02)$ compared to controls. In contrast, the duration of struggling was similar in both groups $(p=0.10)$.

Forced swimming caused a significant increase in the local release of various amino acids as reflected by a higher concentration of aspartate, glutamate, and taurine in microdialysates sampled during perfusion periods 3 and 4 , compared to dialysates sampled under basal conditions (Figure 4). In contrast, the release of arginine and GABA was unaffected by exposure to swim stress.

Local administration of the OXT receptor antagonist into the CeA did not alter basal release patterns of any of the amino acids measured within the amygdala. In contrast, the forced swimming-induced release of the excitatory amino acids glutamate (factor group $\times$ time, $\mathrm{F}_{4,32}=4.92, p=0.003$ ) and aspartate $\left(\mathrm{F}_{4,32}=3.24, \quad p=0.02\right)$ was significantly increased. The release of taurine, arginine, and GABA within the $\mathrm{CeA}$ was not influenced by local administration of the OXT receptor antagonist during swimming.

\section{DISCUSSION}

The results of the present study demonstrate that OXT release within the CeA of male rats can be monitored using microdialysis in conjunction with a highly sensitive radioimmunoassay both under stimulated and basal conditions. OXT is released within the CeA in response to an ethologically relevant stimulus, as exposure of male rats to swim stress caused an increase in neuropeptide release. Furthermore, administration of an OXT receptor antagonist via inverse microdialysis into the $\mathrm{CeA}$ with simultaneous behavioral testing revealed that endogenous OXT released within the amygdala is involved in the generation of a passive stress-coping strategy, at least during forced swimming. Possible mechanisms of these behavioral actions might include the regulation of local release patterns of relevant excitatory amino acids, as OXT receptor blockade

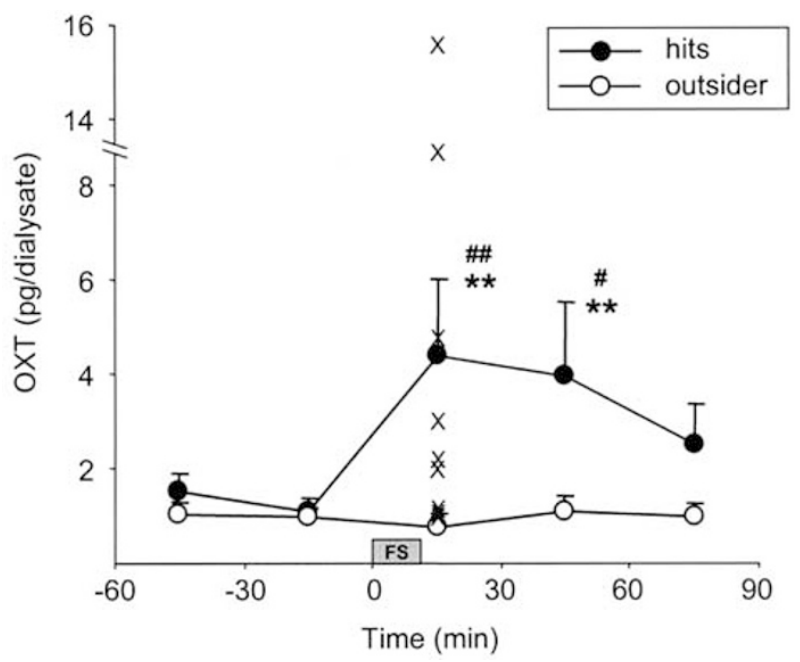

Figure 2 Effects of forced swimming on OXT content in five consecutive 30-min dialysates collected within ( $n=9$, black circles) or outside ( $n=4$, white circles) the CeA of conscious, freely moving male rats under basal conditions and during forced swimming (FS, gray shaded bar; $10 \mathrm{~min}$ at $20^{\circ} \mathrm{C}$ ). Data are expressed as means + SEM. Individual data points at the time point during forced swimming are indicated by $X$. *** $p<0.0$ I vs dialysates I and $2 ; \# p<0.05$, \#\#p<0.0I vs respective value in the outsider group (two-way ANOVA followed by Newman-Keuls post hoc test)
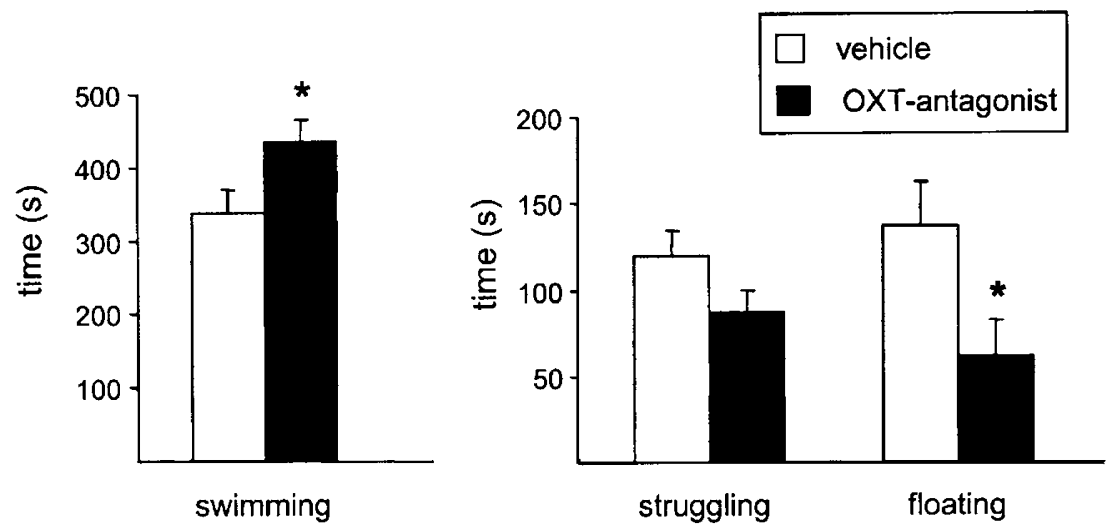

Figure 3 Effect of an OXT receptor antagonist applied bilaterally into the CeA on active and passive stress-coping parameters during I0-min forced swimming as indicated by the time the animals spent swimming, struggling, and floating (means + SEM). Either vehicle (Ringer's solution, $n=5)$ or the OXT receptor antagonist (des-Gly- $\left.\mathrm{NH}_{2} \mathrm{~d}\left(\mathrm{CH}_{2}\right)_{5}\left(\mathrm{Tyr}(\mathrm{Me})^{2} \mathrm{Thr}^{4}\right) \mathrm{OVT} ; 10 \mu \mathrm{g} / \mathrm{ml}, 3.3 \mu \mathrm{l} / \mathrm{min}, n=6\right)$ was applied bilaterally into the CeA via inverse microdialysis. $* p<0.05$ vs vehicle-treated controls (Mann-Whitney $U$-test). 

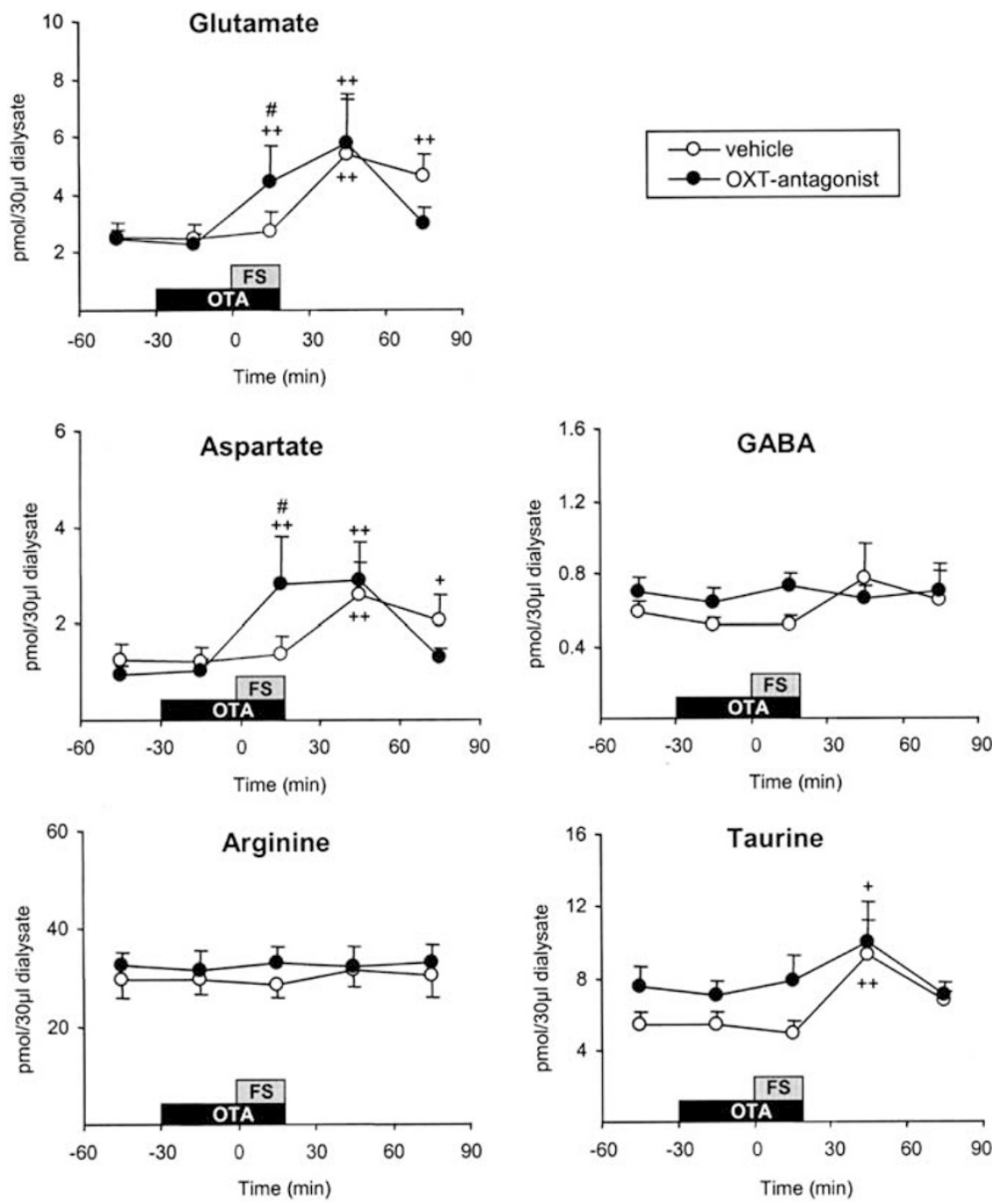

Figure 4 Concentration of the amino acids glutamate, aspartate, GABA, arginine, and taurine in 30-min microdialysates (means + SEM) obtained from the $\mathrm{CeA}$ of male rats before, during, and after forced swimming (FS, gray shaded bars). During dialysis periods 2 and 3, either vehicle (Ringer's solution, open circles, $n=4)$ or Ringer's solution containing the OXT receptor antagonist des-Gly- $\mathrm{NH}_{2} \mathrm{~d}\left(\mathrm{CH}_{2}\right)_{5}\left(\mathrm{Tyr}(\mathrm{Me})^{2} \mathrm{Thr}{ }^{4}\right) \mathrm{OVT}(\mathrm{OTA}$, black circles, $n=6)$ was used as perfusion fluid. At the beginning of dialysis period 3, the animals were forced to swim for 10 min $\left(20^{\circ} \mathrm{C}\right)$. $+p<0.05,++p<0.0$ I vs basal; \# $p<0.05$ vs respective value in the vehicle-treated controls (two-way ANOVA followed by Fisher's LSD post hoc test).

also increased the stress-induced release of glutamate and aspartate within the CeA.

It is of note that we were able to detect OXT content in microdialysis samples from the CeA, thus allowing for the first time monitoring of the dynamics of local release patterns (Landgraf and Neumann, 2004) and, specifically, the rise in local nonapeptide in response to stress. Since neuropeptides such as OXT only become biologically active after their release into the extracellular space, attempts to measure intracerebral release focus on approaches that are able to determine concentrations and their fluctuations in the extracellular fluid including intracerebral microdialysis. In this way, OXT release has been monitored in several in vivo studies mainly focusing on somato-dendritic release within the hypothalamic SON and PVN under physiological or pharmacological conditions (Moos et al, 1989; Neumann et al, 1993a,b; Nishioka et al, 1998; Ludwig et al, 2002; Wigger and Neumann, 2002; for review, see Landgraf and
Neumann, 2004). In contrast, only a few reports are available demonstrating fluctuations in the release of OXT within extrahypothalamic brain regions like the septum (Neumann and Landgraf, 1989; Landgraf et al, 1991; Ebner et al, 2000) or the olfactory bulb (Kendrick et al, 1988a, b) under physiologically relevant conditions. Sparse innervation and consequently low concentrations of OXT in the extracellular fluid make quantification of local in vivo release within these limbic brain regions difficult. However, with respect to stress coping, we could previously demonstrate OXT release within the septum of male rats in response to social defeat (Ebner et al, 2000). Furthermore, within the $\mathrm{CeA}$ of virgin and lactating female rats, OXT release could be monitored under basal conditions (Bosch et al, 2004), although exposure to a psycho-social stressor (maternal defeat) did not elevate intra-amygdaloid release of OXT (Bosch et al, 2004). Thus, the present study provides the first evidence that a relevant stressor like 
forced swimming triggers local OXT release in males. Interestingly, exposure to swimming - a complex physiological and psychological stressor (Abel, 1994) - triggers not only OXT secretion into blood (Lang et al, 1983, Wotjak et al, 1998) but also OXT synthesis (Wotjak et al, 2001) and release within the SON and PVN in males (Wotjak et al, 1998) and females (Wigger and Neumann, 2002). Thus, the question arises as to the origin of OXT measured in dialysates from the CeA. The fact that the stress-induced increase in extracellular OXT concentration was restricted to the area of the $\mathrm{CeA}$, but was not found outside that region (Figure 2), excludes the possibility of unspecific diffusion, for example, from hypothalamic nuclei discussed before (Landgraf and Neumann, 2004). Furthermore, our finding is consistent with immunohistochemical studies demonstrating a relatively dense plexus of OXT-containing terminals mainly in the central amygdaloid nucleus (Sofroniew, 1983). These fibers have been found to originate predominantly from the PVN, since OXT fiber density decreases dramatically in all OXT projection sites after lesioning this hypothalamic area (De Vries and Buijs, 1983; Van Leeuwen and Caffe, 1983).

With respect to the possible physiological significance of OXT released within the amygdala, it is important to mention that OXT receptors and OXT-binding sites have been localized throughout the amygdala, with particularly high density in its central part (Elands et al, 1988; Tribollet et al, 1988; Kremarik et al, 1993; Yoshimura et al, 1993; Condés-Lara et al, 1994). In general, in contrast to females, physiological functions of brain OXT are less well documented in males. Various studies imply a critical role for OXT in the regulation of physiological and behavioral processes related to learning/memory, emotionality, and stress coping (Argiolas and Gessa, 1991; Richard et al, 1991; Engelmann et al, 1996, Gimpl and Fahrenholz, 2001; Landgraf and Neumann, 2004). Here, we applied the selective OXT receptor antagonist bilaterally into the CeA to investigate the receptor-mediated behavioral effects of OXT during forced swimming. Previous studies proved this approach useful for investigating the behavioral significance of endogenously released neuropeptides such as vasopressin within the amygdala (Ebner et al, 2002). In the present study, blockade of the oxytocinergic neurotransmission/neuromodulation in the amygdala by local antagonist treatment significantly pronounced the active stress-coping style with prolonged swimming and reduced floating behavior (Figure 3), which suggests that endogenous OXT is involved in the promotion of a rather passive stress-coping strategy. This is in line with neurochemical and behavioral studies demonstrating a pivotal role of OXT within the $\mathrm{CeA}$ in the regulation of emotional behaviors, as it acts as an endogenous anxiolytic (Bale et al, 2001; Neumann, 2002), and also demonstrating that local OXT inhibits the stress-induced activity of the male HPA axis (Neumann et al, 2000b). Thus, summarizing these behavioral and physiological effects within the CeA, our data suggest that OXT may contribute to general sedation to prevent excessive stress responses (Windle et al, 2004).

Although precise information regarding the penetration and diffusion rate of the infused antagonist compound via inverse microdialysis are lacking, the OXT antagonist most likely diffused to neighboring subregions of the amygdala. In this context, it is important to note that the amygdala is a structure of anatomically distinct subsystems representing a complex inter-related network involved in the processing of various physiological functions (Adelheid et al, 1995; Pitkänen et al, 1997). OXT-binding sites have been detected in several subregions of the amygdala (Barberis and Tribollet, 1996; Gimpl and Fahrenholz, 2001), although a number of studies (see above) suggested that the CeA is a specific target region for OXT. The behavioral effects observed may be based on the ability of OXT to alter the electrical activity of amygdala neurons. Condés-Lara et al (1994) showed that, in vivo, nearly $50 \%$ of recorded neurons in the amygdala, mostly in the central nucleus, responded to iontophoretic application of OXT by a change of their firing rate discharge, suggesting the presence of functional receptors.

Neuropeptides like OXT may function, in general, as intracerebral neuromodulators rather than as neurotransmitters (Landgraf and Neumann, 2004), and this may hold true also within the amygdala. In this context, it is of note that other neurotransmitter systems were modulated by locally released OXT. Both excitatory and inhibitory amino acids, among them glutamate and GABA, which provide the principal sources of synaptic transmission in the amydala, are possible candidates. Interestingly, in response to swim stress, the release of glutamate, aspartate, and taurine was found to be elevated within the CeA (Figure 4), whereas local GABA release remained unchanged. As local treatment with the OXT receptor antagonist during forced swimming further enhanced the intra-amygdaloid release of glutamate and aspartate compared to Ringer-treated control rats (Figure 4), an inhibitory influence of locally released OXT on these excitatory neurotransmitter systems within the amygdala is suggested during a physiological stress response. However, this modulatory effect was restricted to the stimulated state during stressor exposure (and elevated local release of OXT), but could not be found under basal conditions. Thus, the functional interaction between OXT and these neurotransmitter systems seems to be highly relevant for stress coping and is in accordance with the finding of endogenous OXT promoting a rather passive behavioral coping style within the amygdala. Since excitatory amino acid neurotransmission within the amygdala is stress sensitive (Singewald et al, 2000) and seems to be critically involved in behavioral regulation, including the mediation and expression of stress- and anxiety-related behaviors (Adamec et al, 1999; Walker et al, 2002), it is tempting to speculate that the interplay between these systems within the amygdala modulates the behavioral responses to acute stress. These data are also consistent with the demonstration of reduced depolarization-induced glutamate and aspartate release from SON tissue after OXT application (Currás-Collazo et al, 2003). In contrast, the release of the inhibitory amino acids GABA and taurine was not affected by local OXT antagonist treatment, which is interesting, as swim stress itself did not alter the local release of GABA.

However, as various studies implicate a pivotal role for the GABAergic system within the amygdala in emotional behavior (Davis et al, 1994; Shekhar et al, 2003), it is important to exclude technical limitations of our study. We 
were able to monitor GABA concentrations in the extracellular fluid under basal conditions, which were clearly above the detection limit of the HPLC. Thus, any stress- or treatment-related alterations in local GABA release patterns would have been detected.

In summary, the results of the present study demonstrate that under forced swimming conditions OXT is released within the $\mathrm{CeA}$ of male rats. Furthermore, such locally released OXT modulates the behavioral stress response as it promotes a passive stress-coping style, probably by its inhibitory effects on the local release of the excitatory amino acids glutamate and aspartate.

\section{ACKNOWLEDGEMENTS}

We are grateful to Professor R Landgraf for radioimmunological quantification of OXT. We thank $M$ Fuchs, $\mathrm{P}$ Lörscher, and S Karg for excellent technical help. This work was supported by the Deutsche Forschungsgemeinschaft (DFG, IDN; Ne465).

\section{REFERENCES}

Abel EL (1994). A further analysis of physiological changes in rats in the forced swim test. Physiol Behav 56: 795-800.

Adamec RE, Burton P, Shallow T, Budgell J (1999). Unilateral block of NMDA receptors in the amygdala prevents predator stressinduced lasting increases in anxiety-like behavior and unconditioned startle-effective hemisphere depends on the behavior. Physiol Behav 65: 739-751.

Adelheid GF, De Olmos JS, Beltramino CA (1995). Amygdala and extended amygdala. In: Paxinos G (ed). The Rat Nervous System. Academic Press: New York. pp 495-578.

Aggleton JP (1992). The Amygdala. Neurochemical Aspects of Emotion, Memory and Mental Dysfunction. John Wiley-Liss and Sons: New York.

Argiolas A, Gessa GL (1991). Central functions of oxytocin. Neurosci Biobehav Rev 15: 217-231.

Bale TL, Davis AM, Auger AP, Dorsa DM, McCarthy MM (2001). CNS region-specific oxytocin receptor expression: importance in regulation of anxiety and sex behavior. J Neurosci 21: 2546-2552.

Barberis C, Tribollet E (1996). Vasopressin and oxytocin receptors in the central nervous system. Crit Rev Neurobiol 10: 119-154.

Bosch OJ, Krömer SA, Brunton PJ, Neumann ID (2004). Release of oxytocin in the hypothalamic paraventricular nucleus, but not central amygdala or lateral septum in lactating residents and virgin intruders during maternal defence. Neuroscience 124: $439-448$

Callahan MF, Kirby RF, Cunningham JT, Eskridge-Sloop SL, Johnson AK, McCarty R et al (1989). Central oxytocin systems may mediate a cardiovascular response to acute stress in rats. Am J Physiol 256: H1369-H1377.

Condés-Lara M, Veinante P, Rabai M, Freund-Mercier MJ (1994). Correlation between oxytocin neuronal sensitivity and oxytocinbinding sites in the amygdala of the rat: electrophysiological and histoautoradiographic study. Brain Res 637: 277-286.

Currás-Collazo MC, Gillard ER, Jin J, Pandika J (2003). Vasopressin and oxytocin decrease excitatory amino acid release in adult rat supraoptic nucleus. J Neuroendocrinol 15: 182-190.

Davis M, Rainnie D, Cassell M (1994). Neurotransmission in the rat amygdala related to fear and anxiety. Trends Neurosci 17: 208-214.

De Vries GJ, Buijs RM (1983). The origin of the vasopressinergic and oxytocinergic innervation of the rat brain with special reference to the lateral septum. Brain Res 273: 307-317.
Ebner K, Wotjak CT, Holsboer F, Landgraf R, Engelmann M (1999). Vasopressin released within the septal brain area during swim stress modulates the behavioural stress response in rats. Eur J Neurosci 11: 997-1002.

Ebner K, Wotjak CT, Landgraf R, Engelmann M (2000). A single social defeat experience selectively stimulates the release of oxytocin, but not vasopressin, within the septal brain area of male rats. Brain Res 872: 87-92.

Ebner K, Wotjak CT, Landgraf R, Engelmann M (2002). Forced swimming triggers vasopressin release within the amygdala to modulate stress-coping strategies in rats. Eur J Neurosci 15: 384-388.

Elands J, Beetsma A, Barberis C, de Kloet ER (1988). Topography of the oxytocin receptor system in rat brain: an autoradiographical study with a selective radioiodinated oxytocin antagonist. J Chem Neuroanat 1: 293-302.

Engelmann M, Ebner K, Landgraf R, Holsboer F, Wotjak CT (1999). Emotional stress triggers intrahypothalamic but not peripheral release of oxytocin in male rats. J Neuroendocrinol 11: 867-872.

Engelmann M, Ludwig M, Landgraf R (1992). Microdialysis administration of vasopressin and vasopressin antagonists into the septum during pole-jumping behavior in rats. Behav Neural Biol 58: 51-57.

Engelmann M, Wotjak CT, Neumann ID, Ludwig M, Landgraf R (1996). Behavioral consequences of intracerebral vasopressin and oxytocin: focus on learning and memory. Neurosci Biobehav Rev 20: 341-358.

Gimpl G, Fahrenholz F (2001). The oxytocin receptor system: structure, function, and regulation. Physiol Rev 81: 629-683.

Gray TS (1996). Amygdala: role in autonomic and neuroendocrine responses to stress. In: McCubbin JB, Kaufmann PG, Nemeroff CB (eds). Stress, Neuropeptides and Systemic Disease. Academic Press: San Diego. pp 37-53.

Iványi T, Wiegant VM, De Wied D (1991). Differential effects of emotional and physical stress on the central and peripheral secretion of neurohypophysial hormones in male rats. Life Sci 48: $1309-1316$.

Kasting NW (1988). Simultaneous and independent release of vasopressin and oxytocin in the rat. Can J Physiol Pharmacol 66: $22-26$.

Kendrick KM, Keverne EB, Chapman C, Baldwin BA (1988a). Microdialysis measurement of oxytocin, aspartate, gammaaminobutyric acid and glutamate release from the olfactory bulb of the sheep during vaginocervical stimulation. Brain Res 442: 171-174.

Kendrick KM, Keverne EB, Chapman C, Baldwin BA (1988b). Intracranial dialysis measurement of oxytocin, monoamine and uric acid release from the olfactory bulb and substantia nigra of sheep during parturition, suckling, separation from lambs and eating. Brain Res 439: 1-10.

Kremarik P, Freund-Mercier MJ, Stoeckel ME (1993). Histoautoradiographic detection of oxytocin- and vasopressinbinding sites in the telencephalon of the rat. J Comp Neurol 333: 343-359.

Landgraf R, Kubota M, Holsboer F, Wotjak CT (1995). Release of vasopressin and oxytocin within the brain and into blood: microdialysis and antisense targeting. In: Saito T, Kurokawa K, Yoshida S (eds). Neurohypophysis: Recent Progress of Vasopressin and Oxytocin Research. Elsevier: Amsterdam. pp 243-256.

Landgraf R, Neumann ID (2004). Vasopressin and oxytocin release within the brain: a dynamic concept of multiple and variable modes of neuropeptide communication. Front Neuroendocrinol, (in press).

Landgraf R, Neumann ID, Pittman QJ (1991). Septal and hippocampal release of vasopressin and oxytocin during late pregnancy and parturition in the rat. Neuroendocrinology 54: $378-383$ 
Lang RE, Heil J, Ganten D, Hermann K, Unger T, Rascher W (1983). Oxytocin unlike vasopressin is a stress hormone in the rat. Neuroendocrinology 37: 314-316.

Lightman SL, Windle RJ, Wood SA, Kershaw YM, Shanks N, Ingram CD (2001). Peripartum plasticity within the hypothalamo-pituitary-adrenal axis. Prog Brain Res 133: 111-129.

Ludwig M, Sabatier N, Bull PM, Landgraf R, Dayanithi G, Leng G (2002). Intracellular calcium stores regulate activity-dependent neuropeptide release from dendrites. Nature 418: 85-89.

Maren S (1996). Synaptic transmission and plasticity in the amygdala. An emerging physiology of fear conditioning circuits. Mol Neurobiol 13: 1-22.

Moos F, Poulain DA, Rodriguez F, Guerne Y, Vincent JD, Richard $P$ (1989). Release of oxytocin within the supraoptic nucleus during the milk ejection reflex in rats. Exp Brain Res 76: 593-602.

Neumann I, Ludwig M, Engelmann M, Pittman QJ, Landgraf R (1993a). Simultaneous microdialysis in blood and brain: oxytocin and vasopressin release in response to central and peripheral osmotic stimulation and suckling in the rat. Neuroendocrinology 58: 637-645.

Neumann I, Russell JA, Landgraf R (1993b). Oxytocin and vasopressin release within the supraoptic and paraventricular nuclei of pregnant, parturient and lactating rats: a microdialysis study. Neuroscience 53: 65-75.

Neumann ID (2001). Alterations in behavioral and neuroendocrine stress coping strategies in pregnant, parturient and lactating rats. Prog Brain Res 133: 143-152.

Neumann ID (2002). Involvement of the brain oxytocin system in stress coping: interactions with the hypothalamo-pituitaryadrenal axis. Prog Brain Res 139: 147-162.

Neumann ID, Kromer SA, Toschi N, Ebner K (2000b). Brain oxytocin inhibits the (re)activity of the hypothalamo-pituitaryadrenal axis in male rats: involvement of hypothalamic and limbic brain regions. Regul Peptides 96: 31-38.

Neumann ID, Landgraf R (1989). Septal and hippocampal release of oxytocin, but not vasopressin, in the conscious lactating rat during suckling. J Neuroendocrinol 1: 305-308.

Neumann ID, Torner L, Wigger A (2000a). Brain oxytocin: differential inhibition of neuroendocrine stress responses and anxiety-related behaviour in virgin, pregnant and lactating rats. Neuroscience 95: 567-575.

Nishioka T, Anselmo-Franci JA, Li P, Callahan MF, Morris M (1998). Stress increases oxytocin release within the hypothalamic paraventricular nucleus. Brain Res 781: 56-60.

Paxinos G, Watson C (1998). The Rat Brain in Stereotaxic Coordinates. Academic Press: Sydney.

Pitkänen A, Savander V, LeDoux JE (1997). Organization of intraamygdaloid circuitries in the rat: an emerging framework for understanding functions of the amygdala. Trends Neurosci 20: 517-523.

Richard P, Moos F, Freund-Mercier MJ (1991). Central effects of oxytocin. Physiol Rev 71: 331-370.

Sah P, Faber ES, Lopez De Armentia M, Power J (2003). The amygdaloid complex: anatomy and physiology. Physiol Rev 83: 803-834.
Shekhar A, Sajdyk TJ, Gehlert DR, Rainnie DG (2003). The amygdala, panic disorder, and cardiovascular responses. Ann NY Acad Sci 985: 308-325.

Singewald N, Kouvelas D, Mostafa A, Sinner C, Philippu A. (2000). Release of glutamate and GABA in the amygdala of conscious rats by acute stress and baroreceptor activation: differences between SHR and WKY rats. Brain Res 864: 138-141.

Singewald N, Zhou GY, Schneider C (1995). Release of excitatory and inhibitory amino acids from the locus coeruleus of conscious rats by cardiovascular stimuli and various forms of acute stress. Brain Res 704: 42-50.

Sofroniew MV (1983). Vasopressin and oxytocin in the mammalian brain and spinal cord. Trends Neurosci 6: 467-472.

Tribollet E, Barberis C, Jard S, Dubois-Dauphin M, Dreifuss JJ (1988). Localization and pharmacological characterization of high affinity binding sites for vasopressin and oxytocin in the rat brain by light microscopic autoradiography. Brain Res 442: 105-118.

Van Leeuwen F, Caffe R (1983). Vasopressin-immunoreactive cell bodies in the bed nucleus of the stria terminalis of the rat. Cell Tissue Res 228: 525-534.

Walker DL, Rattiner LM, Davis M (2002). Group II metabotropic glutamate receptors within the amygdala regulate fear as assessed with potentiated startle in rats. Behav Neurosci 116: 1075-1083.

Wigger A, Neumann ID (2002). Endogenous opioid regulation of stress-induced oxytocin release within the hypothalamic paraventricular nucleus is reversed in late pregnancy: a microdialysis study. Neuroscience 112: 121-129.

Windle RJ, Kershaw YM, Shanks N, Wood SA, Lightman SL, Ingram CD (2004). Oxytocin attenuates stress-induced c-fos mRNA expression in specific forebrain regions associated with modulation of hypothalamo-pituitary-adrenal activity. J Neurosci 24: 2974-2982.

Windle RJ, Shanks N, Lightman SL, Ingram CD (1997). Central oxytocin administration reduces stress-induced corticosterone release and anxiety behavior in rats. Endocrinology 138: 2829-2834.

Wotjak CT, Ganster J, Kohl G, Holsboer F, Landgraf R, Engelmann M (1998). Dissociated central and peripheral release of vasopressin, but not oxytocin, in response to repeated swim stress: new insights into the secretory capacities of peptidergic neurons. Neuroscience 4: 1209-1222.

Wotjak CT, Kubota M, Liebsch G, Montkowski A, Holsboer F, Neumann I et al (1996). Release of vasopressin within the rat paraventricular nucleus in response to emotional stress: a novel mechanism of regulating adrenocorticotropic hormone secretion? J Neurosci 16: 7725-7732.

Wotjak CT, Naruo T, Muraoka S, Simchen R, Landgraf R, Engelmann M (2001). Forced swimming stimulates the expression of vasopressin and oxytocin in magnocellular neurons of the rat hypothalamic paraventricular nucleus. Eur J Neurosci 13: 2273-2281.

Yoshimura R, Kiyama H, Kimura T, Araki T, Maeno H, Tanizawa $O$ et al (1993). Localization of oxytocin receptor messenger ribonucleic acid in the rat brain. Endocrinology 133: 1239-1246. 